\title{
Structural study of supercritical water. III. Rotational dynamics
}

$\operatorname{AUTHOR}(S)$ :

Matubayasi, N; Nakao, N; Nakahara, M

\section{CITATION:}

Matubayasi, N ...[et al]. Structural study of supercritical water. III. Rotational dynamics. JOURNAL OF CHEMICAL PHYSICS 2001, 114(9): 4107-4115

\section{ISSUE DATE:}

2001-03-01

URL:

http://hdl.handle.net/2433/50352

\section{RIGHT:}

Copyright 2001 American Institute of Physics. This article may be downloaded for personal use only. Any other use requires prior permission of the author and the American Institute of Physics. 


\title{
Structural study of supercritical water. III. Rotational dynamics
}

\author{
Nobuyuki Matubayasi, ${ }^{\text {a) }}$ Naoko Nakao, and Masaru Nakahara ${ }^{a)}$ \\ Institute for Chemical Research, Kyoto University, Uji, Kyoto 611-0011, Japan
}

(Received 18 August 2000; accepted 6 November 2000)

\begin{abstract}
The rotational dynamics of water in super- and subcritical conditions is investigated by measuring the spin-lattice relaxation time $T_{1}$ of heavy water $\left(\mathrm{D}_{2} \mathrm{O}\right)$. The experimentally determined $T_{1}$ is shown to be governed by the quadrupolar relaxation mechanism even in the supercritical conditions and to provide the second-order reorientational correlation time $\tau_{2 R}$ of the $\mathrm{O}-\mathrm{D}$ axis of a single water molecule. It is then found that while $\tau_{2 R}$ decreases rapidly with the temperature on the liquid branch of the saturation curve, it remains on the order of several tens of femtoseconds when the density is varied up to twice the critical at a fixed supercritical temperature of $400{ }^{\circ} \mathrm{C}$. The comparison of $\tau_{2 R}$ with the angular momentum correlation time shows that the rotational dynamics is not diffusive in supercritical water. The dependence of $\tau_{2 R}$ on the hydrogen bonding state is also examined in combination with molecular dynamics simulations, and the effect of the hydrogen bonding on the rotational dynamics in supercritical water is found to be weaker than but to be on the same order of magnitude as that in ambient water on the relative scale. Actually, although $\tau_{2 R}$ is divergent in the limit of zero density, it is observed to increase with the density when the density is above $\sim 1 / 3$ of the critical. (C) 2001 American Institute of Physics. [DOI: 10.1063/1.1336571]
\end{abstract}

\section{INTRODUCTION}

Supercritical water receives much attention recently as a novel and clean medium for chemical processes of environmental and industrial importance. ${ }^{1-5}$ The rate of a chemical process in a fluid medium is determined by the thermodynamics and dynamics of hydration of the chemical species involved in the process. Although the overall behavior of a chemical reaction is governed by the free energy profile along the reaction coordinate, the dynamics of hydration often plays an important role in determining the reaction rate constant and is indispensable for a molecular description of the rate constant. The dynamics of supercritical hydration is inseparably related to the dynamical structure of pure solvent water at the supercritical state. Thus, in order to establish the molecular picture of the hydration dynamics in supercritical water, it is essential to characterize the dynamics of supercritical water as a pure solvent.

The study of the static structures of supercritical water forms a basis for addressing the dynamical counterparts. Previous experimental studies of the static structures focused mainly on the hydrogen bonding structure in supercritical water and revealed that the hydrogen bonding persists even at densities below the critical. ${ }^{6-21}$ While these static studies extract pictures averaged over various hydrogen bonding states, the dynamical studies characterize the interconversion among the microscopic states. The first dynamical study of supercritical water was performed by Jonas et al. ${ }^{22-25}$ a couple of decades before the modern spectroscopic and diffractometric measurements are conducted and the molecular simulation method is intensively employed. ${ }^{6-21,26}$ In their high-temperature and high-pressure studies of $\mathrm{H}_{2} \mathrm{O}$, Jonas et al. determined through proton $\left({ }^{1} \mathrm{H}\right) \mathrm{NMR}$ the translational

${ }^{a)}$ Author to whom correspondence should be addressed. diffusion constant and spin-lattice relaxation time of supercritical water, which represent the two aspects of dynamics, translational and rotational, respectively.

Since the intermolecular interaction of such a hydrogen bonding fluid as water is strongly dependent on the orientation of the molecules involved, the rotational dynamics is considered more suited to probe the local structure of fluid than the translational dynamics. ${ }^{27-29}$ In the classic works by Jonas et al., it was found that the spin-lattice relaxation time of supercritical $\mathrm{H}_{2} \mathrm{O}$ is dominated by the spin-rotation mechanism and provides information about the relaxation time of the angular momentum of a single water molecule. ${ }^{22-25}$ When the local structure is concerned, however, a dynamical study performed in the momentum space is less insightful than the complementary study performed in the configuration space. In other words, it is desirable to adopt an experimental method which probes the rotational dynamics in the configuration space. Microwave spectroscopy is a method to determine the dielectric relaxation time, which characterizes the time scale involved in the collective motion of the total dipole moment of the system. In recent papers by Okada et al., it was reported that the dielectric relaxation time of supercritical water exhibits a sharp inversion as a function of the density. ${ }^{30,31}$ On the other hand, since the hydrogen bonding is still present in supercritical water and the intermolecular correlations are not considered weak, ${ }^{6-21}$ an intuitively appealing picture may be constructed from the dynamical information of a single water molecule. In this work, we determine the reorientational correlation time of a single water molecule in supercritical heavy water $\left(\mathrm{D}_{2} \mathrm{O} ;{ }^{2} \mathrm{H} \equiv \mathrm{D}\right)$ by measuring the deuteron NMR spin-lattice relaxation time. We show that the deuteron spin-lattice relaxation time of supercritical heavy water is governed by the quadrupolar mechanism and provides information about the motion of a single water molecule in the configuration space. 
In order to extract molecular information from an experimentally determined structural quantity, it is often useful to perform a supplementary analysis through computer simulation. In previous works on the static structure of supercritical water, we combined the proton chemical shift measurements with the computer simulations over a wide set of thermodynamic and intramolecular states. ${ }^{6-8} \mathrm{We}$ then examined the validity and limitation of an empirically useful model and determined a quantity which is not easily accessible only from the chemical shift. Since the fluid structure may be examined at desired detail in computer simulation once the intermolecular interactions are given, it is also insightful to perform a dynamical analysis of supercritical water through computer simulation. In this work, we supplement the NMR relaxation measurements with molecular dynamics simulations and extract dynamical information which is consistent with but surpasses that obtained from the NMR measurements.

\section{PROCEDURES}

\section{A. Experiment}

In order to realize a supercritical state of heavy water $\left(\mathrm{D}_{2} \mathrm{O}\right)$, the capillary method described in Refs. 6 and 7 was employed. In this method, water is confined in a sealed capillary made of quartz and the capillary is placed in an NMR sample tube (see Fig. 2 of Ref. 7). The content of water in the capillary uniquely determines the density at supercritical conditions. The density of supercritical heavy water can be conveniently expressed by the packing fraction, which is defined as the ratio of the (liquid) water volume in the capillary to the total volume of the capillary at room temperature. When the packing fraction is $\rho$, the density of heavy water at a supercritical temperature is $1.1 \rho \mathrm{g} / \mathrm{cm}^{3}$, provided that the liquid density is $1.1 \mathrm{~g} / \mathrm{cm}^{3}$ at room temperature. The packing fractions examined in the present work are $\rho=+0.0,0.1$, $0.2,0.3,0.4,0.5$, and 0.6 with errors of less than 0.03 . The expression $\rho=+0.0$ means that the packing fraction is smaller than 0.02 , though water is definitely present in the capillary. We adopt this expression for the lowest packing fraction since it is not possible in our capillary apparatus to identify $\rho$ precisely when $\rho \leq 0.02$. Actually, it is sufficient for the discussion at the end of Sec. III simply to specify that the lowest $\rho$ examined is certainly smaller than 0.1 .

In the following, we express the density of supercritical heavy water in terms of the packing fraction $\rho$. Furthermore, at any thermodynamic state, we express the density as $\rho$ when it is $1.1 \rho \mathrm{g} / \mathrm{cm}^{3}$. This expression is convenient because light water $\left(\mathrm{H}_{2} \mathrm{O}\right)$ with a packing fraction of $\rho$ involves a density of $\rho \mathrm{g} / \mathrm{cm}^{3}$ at a supercritical state and the number densities of $\mathrm{H}_{2} \mathrm{O}$ and $\mathrm{D}_{2} \mathrm{O}$ with the same packing fraction are coincident with each other. Indeed, $\rho$ is simply the ratio of the density at the thermodynamic state of interest to the liquid density at room temperature.

Heavy water (more than $99.9 \%$ D purity) was obtained from CEA (Commissariat a L'Energie Atomique, France) and used without further purification. High-temperature experiments were possible by setting a high-temperature probe in a multipurpose NMR spectrometer (JEOL EX-270 wide- bore type) equipped with an Oxford superconducting magnet $(6.35$ T). See Fig. 1 of Ref. 7 for an overview of our hightemperature probe, which was developed in cooperation with JEOL. In this probe, the temperature was accurate within $5{ }^{\circ} \mathrm{C}$. In the capillary method, a supercritical state is realized only by raising the temperature of the sample. In the relaxation measurements at supercritical states, the temperature was fixed at $400{ }^{\circ} \mathrm{C}$ and the density was taken as a variable. In addition, the measurements were performed at ambient and subcritical temperatures shown in Table I. At these temperatures, water measured was on the liquid branch of the saturation curve and its density was uniquely determined by the temperature. Actually, the densities listed in Table I were taken from the saturation curve of $\mathrm{D}_{2} \mathrm{O}$ when the temperature is below the critical. ${ }^{32}$ Note that the values of the $\mathrm{D}_{2} \mathrm{O}$ density below the critical temperature are close to the corresponding values of the $\mathrm{H}_{2} \mathrm{O}$ density on the saturation curve when the density is expressed in the units of $\mathrm{mol} / \mathrm{cm}^{3}$.

The deuteron spin-lattice relaxation time $T_{1}$ of $\mathrm{D}_{2} \mathrm{O}$ was measured by the inversion-recovery method with the $\pi-t-\pi / 2$ pulse sequence. The resonance frequency of deuteron was $41 \mathrm{MHz}$. In each measurement, the number of delay times $t$ was 20 and the largest $t$ was taken to be more than $7 T_{1}$. The free induction decay signals were accumulated at least twice and the signal-to-noise ratio exceeded 40. At each thermodynamic state, the $T_{1}$ measurement was performed more than five times and was reproducible within $3 \%$. In other words, our precision in the $T_{1}$ value is $3 \%$.

The inner diameter of a capillary employed in the present work is $0.7 \mathrm{~mm}$ when the density $\rho$ is 0.2 or larger and it is $1.5 \mathrm{~mm}$ when $\rho$ is 0.2 or smaller. ${ }^{33}$ At $\rho=0.2$ and $400{ }^{\circ} \mathrm{C}$ the $T_{1}$ values of $\mathrm{D}_{2} \mathrm{O}$ were compared between the two sizes of capillaries and were found to be coincident within our experimental precision. Furthermore, the lengths of the capillaries were between 2 and $3 \mathrm{~cm}$, and the observed $T_{1}$ did not depend on the capillary length when the thermodynamic state was fixed. This shows that the surface effect on the relaxation measurements is negligible for our sample containers. ${ }^{34}$

The dissolution of quartz into water is another possible cause of errors in our $T_{1}$ measurements of $\mathrm{D}_{2} \mathrm{O}$. In an aqueous solution of $\mathrm{SiO}_{2}$, the measured relaxation rate $\left(1 / T_{1}\right)_{\text {meas }}$ consists of three types of relaxation rate. The first one is the rate $\left(1 / T_{1}\right)_{\text {bulk }}$ of bulk water and is the target of our measurements. The second one is the rate $\left(1 / T_{1}\right)_{\text {shell }}$ of the water molecules in the hydration shell of the dissolved form of $\mathrm{SiO}_{2}$. The third rate $\left(1 / T_{1}\right)_{\text {complex }}$ is the contribution of the deuterons which constitute the aqua complex, most probably $\mathrm{D}_{4} \mathrm{SiO}_{4} \cdot{ }^{35}$ When the mole fraction of $\mathrm{SiO}_{2}$ is $x$ in the aqueous solution and the interconversion among the three states of the D nucleus is much faster than the $T_{1}$ process, the measured relaxation rate $\left(1 / T_{1}\right)_{\text {meas }}$ is expressed as

$$
\begin{aligned}
\left(\frac{1}{T_{1}}\right)_{\text {meas }}= & \left(\frac{1}{T_{1}}\right)_{\text {bulk }}+\left(\left(\frac{1}{T_{1}}\right)_{\text {shell }}-\left(\frac{1}{T_{1}}\right)_{\text {bulk }}\right) n x \\
& +\left(\left(\frac{1}{T_{1}}\right)_{\text {complex }}-\left(\frac{1}{T_{1}}\right)_{\text {bulk }}\right) \alpha m x,
\end{aligned}
$$

where $n$ is the hydration number, $\alpha$ is a factor of at most 1 , 
TABLE I. Experimental conditions and results.

\begin{tabular}{cccccc}
\hline \hline & & & \multicolumn{2}{c}{ Reorientational correlation time $\tau_{2 R}(\mathrm{fs})$} \\
\cline { 4 - 5 } Temperature $\left({ }^{\circ} \mathrm{C}\right)$ & Density $(\rho)^{\mathrm{a}}$ & $T_{1}(\mathrm{~s})$ & $\mathrm{QCC}=256 \mathrm{kHz}$ & variable QCC $^{\mathrm{b}}$ & QCC $=308 \mathrm{kHz}$ \\
\hline 30 & 1.00 & $0.50 \pm 0.01$ & $2.1 \times 10^{3}$ & & \\
50 & $0.99^{\mathrm{c}}$ & $0.78 \pm 0.01$ & $1.3 \times 10^{3}$ & & \\
100 & $0.96^{\mathrm{c}}$ & $1.72 \pm 0.03$ & $6.0 \times 10^{2}$ & & \\
150 & $0.92^{\mathrm{c}}$ & $2.82 \pm 0.07$ & $3.7 \times 10^{2}$ & & \\
200 & $0.86^{\mathrm{c}}$ & $4.2 \pm 0.1$ & $2.5 \times 10^{2}$ & & \\
250 & $0.80^{\mathrm{c}}$ & $5.9 \pm 0.2$ & $1.7 \times 10^{2}$ & & \\
300 & $0.71^{\mathrm{c}}$ & $7.3 \pm 0.2$ & $1.4 \times 10^{2}$ & & \\
340 & $0.61^{\mathrm{c}}$ & $9.1 \pm 0.2$ & $1.1 \times 10^{2}$ & & $5.7 \times 10^{1}$ \\
400 & 0.6 & $12.6 \pm 0.1$ & $8.2 \times 10^{1}$ & $6.4 \times 10^{1}$ & $5.3 \times 10^{1}$ \\
400 & 0.5 & $12.7 \pm 0.2$ & $8.1 \times 10^{1}$ & $6.3 \times 10^{1}$ & $5.0 \times 10^{1}$ \\
400 & 0.4 & $13.5 \pm 0.4$ & $7.8 \times 10^{1}$ & $5.8 \times 10^{1}$ & $4.9 \times 10^{1}$ \\
400 & 0.3 & $14.3 \pm 0.3$ & $7.6 \times 10^{1}$ & $5.4 \times 10^{1}$ & $1.9 \times 10^{1}$ \\
400 & 0.2 & $14.6 \pm 0.3$ & $7.1 \times 10^{1}$ & $5.1 \times 10^{1}$ & \\
400 & 0.1 & $15.3 \pm 0.1$ & $6.7 \times 10^{1}$ & $4.8 \times 10^{1}$ & \\
400 & $+0.0^{\mathrm{d}}$ & $3.7 \pm 0.1$ & $2.8 \times 10^{2}$ & $1.9 \times 10^{2}$ & $1.9 \times 10^{2}$ \\
\hline \hline
\end{tabular}

a $\rho$ is the dimensionless ratio of the density at the thermodynamic state of interest to the liquid density at room temperature.

${ }^{\mathrm{b}}$ The variable QCC is determined by assuming that the deviation of the QCC value at a thermodynamic state of interest from that at the dilute gas state is proportional to the corresponding deviation of the average dipole moment of a water molecule calculated by the molecular dynamics simulations.

${ }^{c}$ The liquid branch of the saturation curve.

${ }^{\mathrm{d}}$ The actual value of $\rho$ for the sample with $\rho=+0.0$ is definitely nonzero but smaller than 0.02 . Although the precise value is not determined, it is not necessary for the discussion at the end of Sec. III.

and $m$ is the number of water molecules needed to form one complex molecule. ${ }^{36}$ At ambient conditions, it is observed for both ionic and nonpolar solutes that the relaxation rate $1 / T_{1}$ in the hydration shell differs from that in the bulk within a factor of $\sim 2 .^{29,37}$ It is then natural to consider at super- and subcritical conditions that the relaxation rate $\left(1 / T_{1}\right)_{\text {shell }}$ in the hydration shell of the dissolved form of $\mathrm{SiO}_{2}$ deviates from the rate $\left(1 / T_{1}\right)_{\text {bulk }}$ in the bulk by a factor of less than $\sim 2$. In this case, Eq. (1) shows that a $3 \%$ deviation of the measured rate $\left(1 / T_{1}\right)_{\text {meas }}$ from $\left(1 / T_{1}\right)_{\text {bulk }}$ requires $n x \gtrsim 0.03$ when the contribution of the water molecules in the hydration shell is concerned. Among the thermodynamic states examined in the present work, the solubility of $\mathrm{SiO}_{2}$ is the highest at the density of $\rho=0.6$ and the temperature of $400{ }^{\circ} \mathrm{C}$. Since the solubility of $\mathrm{SiO}_{2}$ is essentially the same between $\mathrm{H}_{2} \mathrm{O}$ and $\mathrm{D}_{2} \mathrm{O}$ when expressed in the units of mole fraction, the mole fraction $x$ of $\mathrm{SiO}_{2}$ in water is $x=4$ $\times 10^{-4}$ at the supercritical state of $\rho=0.6$ and $400{ }^{\circ} \mathrm{C}$ and is smaller at the other thermodynamic states. ${ }^{38}$ Therefore, the water molecules in the hydration shell of the dissolved form of $\mathrm{SiO}_{2}$ affects the relaxation rate by $3 \%$ when the hydration number $n$ is larger than an unphysical value of $\sim 75$. This shows, in turn, that the formation of the hydration shell around the dissolved form of $\mathrm{SiO}_{2}$ does not influence the $T_{1}$ value within our experimental precision. A similar argument can also be employed to rule out the possibility of errors caused by the complex formation expressed by the third term in the right-hand side of Eq. (1).

When a sample capillary is prepared at room temperature, air is contained in the gas phase of the capillary (see Fig. 2 of Ref. 7) and is mixed with water in the hightemperature conditions. Since $20 \%$ of air is oxygen, the concentration of oxygen is less than $10^{-2} \mathrm{~mol} / \mathrm{l}$ in the one-phase region and this amount of paramagnetic oxygen may shorten the spin-lattice relaxation spuriously. In order to examine the possibility of the spurious shortening, we also conducted the $T_{1}$ measurements after oxygen was purged from the air in the capillary and substituted with nitrogen. The measurements were performed at a density of $\rho=0.1$ and a supercritical temperature of $400{ }^{\circ} \mathrm{C}$. It was then found within our experimental precision that the measured deuteron $T_{1}$ for the samples without oxygen are coincident with those with oxygen in air. Thus, since the oxygen concentration in a sample with $\rho \geqslant 0.2$ is lower than that in a sample with $\rho=0.1$, the effect of oxygen is negligible when $\rho \geqslant 0.1$. Oxygen was purged for the sample with $\rho=+0.0$.

\section{B. Simulation}

The molecular dynamics simulation was performed for both light water $\left(\mathrm{H}_{2} \mathrm{O}\right)$ and heavy water $\left(\mathrm{D}_{2} \mathrm{O}\right)$. As seen in Sec. II A, the densities of these two water can be conveniently expressed by the packing fraction $\rho$. The supercritical states simulated in the present work mostly correspond to those treated experimentally and are specified by densities of $\rho=0.02,0.05,0.10,0.20,0.30,0.40,0.50$, and 0.60 and a temperature of $400{ }^{\circ} \mathrm{C} .{ }^{39}$ For reference, we also simulated an ambient condition of $\rho=1.00$ and $30^{\circ} \mathrm{C}$.

The TIP4P-FQ model was adopted as the intermolecular potential function between water molecules. ${ }^{40}$ In this water model, the distribution of the partial charges in a water molecule fluctuates in response to the configuration of the other water molecules. The periodic boundary condition was employed and the Ewald summation was implemented to handle the electrostatic potentials. The screening parameter was set to $5 / L$, where $L$ is the length of the unit cell, and 256 
reciprocal lattice vectors were used. In order to perform an efficient and stable molecular dynamics simulation, it is desirable to employ a time-reversible and area-preserving algorithm. This type of algorithm appropriate for such an asymmetric molecule as water was developed by Dullweber $e t a l$. and subsequently and independently by us. ${ }^{41,42}$ In this work, our version of the algorithm was used with a time step of $1 \mathrm{fs}$ by locating 256 water molecules in a cubic unit cell, and the molecular dynamics was carried out for 100 ps at each state of interest.

The self-consistent equation for determining the partial charge distribution in the TIP4P-FQ model was solved by the iterative scheme. When the partial charge distribution at the previous configuration is used at the initial step of iteration, however, the distribution at the current configuration depends on that at the previous configuration and the timereversibility of the molecular dynamics algorithm is violated. This difficulty can be easily circumvented by setting the partial charge distribution at the initial step of iteration to be the one independent of the instantaneous configuration, for example, the predetermined average distribution. It was seen in the present work, nevertheless, that the quantities of interest calculated when the partial charge distribution at the previous configuration is utilized to initialize the iteration are not different from those calculated when the predetermined average distribution is utilized. Thus, we adopted the partial charge distribution at the previous configuration as the distribution at the initial step of iteration for the current configuration.

\section{RESULTS AND DISCUSSION}

In Fig. 1(a), we show the evolution of the deuteron spectrum with the delay time for a sample with the lowest density of $\rho=+0.0$ at a supercritical temperature of $400{ }^{\circ} \mathrm{C}$. It is indeed seen that the signal-to-noise ratio is large enough to determine the spin-lattice relaxation time $T_{1}$. At the other thermodynamic states, the acquisition of the spectra is easier than at $\rho=+0.0$, in proportion to the density involved. The magnetizations $M(t)$ extracted from Fig. 1(a) and those at $\rho=0.1$ and $400{ }^{\circ} \mathrm{C}$ are plotted in Fig. 1(b) as functions of the delay time $t$. The exponential decay to the equilibrium value $M_{0}$ is evident and $T_{1}$ can be accurately evaluated. Figure 1(b) also shows the inversion recovery at an ambient liquid state with a density of $\rho=1.0$ and a temperature of $30^{\circ} \mathrm{C}$. The decay to the equilibrium value at the ambient state seems almost instant when compared to the decay at the supercritical state with $\rho=0.1$. In other words, when $\rho$ is not too small, $T_{1}$ at a supercritical state is longer by an order of magnitude than that in the ambient state and a $T_{1}$ measurement takes a much longer time in the supercritical state.

In Table I, we show the experimentally determined $T_{1}$ of heavy water over the thermodynamic states treated in this work. It is seen that while $T_{1}$ increases rapidly with the temperature on the liquid branch of the saturation curve, it remains on the same order of magnitude against the density variation between $\rho=0.1$ and 0.6 at a fixed temperature of $400{ }^{\circ} \mathrm{C}$. In order to elucidate the molecular-level dynamics of supercritical water, it is necessary to determine the relaxation mechanism governing $T_{1}$. In general, the dipolar mechanism
Matubayasi, Nakao, and Nakahara
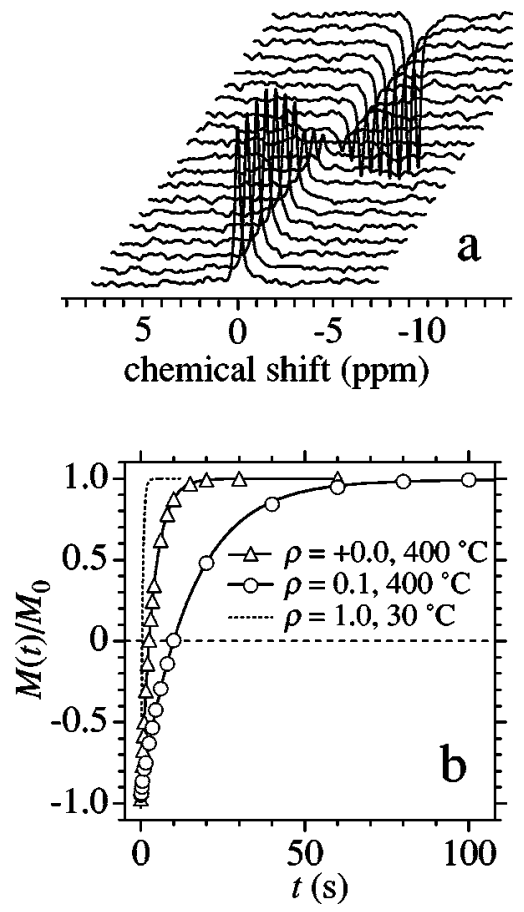

FIG. 1. (a) The evolution of the deuteron spectrum of $\mathrm{D}_{2} \mathrm{O}$ with the delay time for a sample with a density of $\rho=+0.0$ at a supercritical temperature of $400{ }^{\circ} \mathrm{C}$. (b) The normalized magnetization $M(t) / M_{0}$ as a function of the delay time $t$ at $\rho=+0.0$ and 0.1 and $400{ }^{\circ} \mathrm{C}$. The experimentally determined $M(t) / M_{0}$ and its exponential fit are represented by the open symbols and the solid line, respectively. $M(t) / M_{0}$ at an ambient liquid state with a density of $\rho=1.0$ and a temperature of $30^{\circ} \mathrm{C}$ is also shown in (b) by the dotted line.

is overwhelmed by the quadrupolar mechanism for a deuteron. ${ }^{43}$ Furthermore, at ambient conditions, the quadrupolar mechanism controls $T_{1}$ since the spin-rotation mechanism is negligible. At high-temperature conditions, however, the relaxation of the angular momentum of a molecule becomes slow and careful treatment may be needed for the competition between the quadrupolar and spin-rotation mechanisms. We estimate the contribution of the spinrotation mechanism to the deuteron $T_{1}$ of supercritical heavy water $\left(\mathrm{D}_{2} \mathrm{O}\right)$ on the basis of the proton $T_{1}$ of supercritical light water $\left(\mathrm{H}_{2} \mathrm{O}\right)$ determined by Jonas et al. ${ }^{22,23}$ Let $1 / T_{1, \mathrm{sr}}^{h}$ and $1 / T_{1, \text { sr }}^{l}$ be the contributions of the spin-rotation mechanism to the $1 / T_{1}$ of heavy and light water, respectively. According to Ramsey, the spin-rotation tensor is proportional to the magnetogyric ratio and inversely to the moment of inertia for a series of molecules in which the electronic structure is not different and the molecular vibration is not significant. ${ }^{44}$ It is then easy to see that

$$
\frac{1 / T_{1, \mathrm{sr}}^{h}}{1 / T_{1, \mathrm{sr}}^{l}} \approx \frac{I^{l} \tau_{J}^{h}}{I^{h} \tau_{J}^{l}}\left(\frac{\gamma^{h}}{\gamma^{l}}\right)^{2}
$$

where $I^{h}, \gamma^{h}$, and $\tau_{J}^{h}$ are, respectively, the average moment of inertia of $\mathrm{D}_{2} \mathrm{O}$, the deuteron magnetogyric ratio, and the correlation time of the angular momentum of $\mathrm{D}_{2} \mathrm{O}$, and $I^{l}$, $\gamma^{l}$, and $\tau_{J}^{l}$ are the corresponding quantities for $\mathrm{H}_{2} \mathrm{O}$. The computer simulations provide a reasonable estimate of $\tau_{J}^{h} / \tau_{J}^{l}$. In Fig. 2(a), we show $\tau_{J}^{h}$ and $\tau_{J}^{l}$ at the supercritical conditions simulated. The angular momentum correlation time $\tau_{J}^{l}$ of $\mathrm{H}_{2} \mathrm{O}$ calculated by employing the TIP4P-FQ 

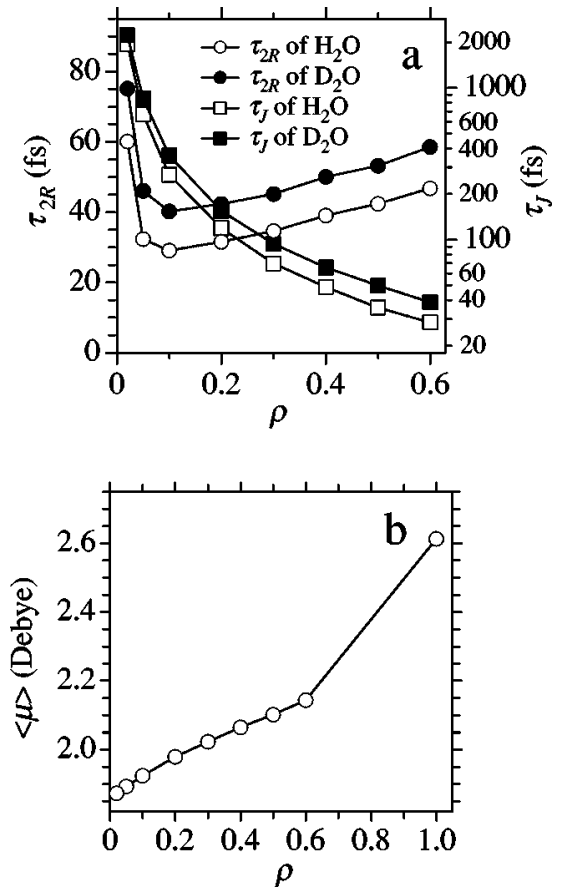

FIG. 2. (a) The reorientational correlation time $\tau_{2 R}$ and the angular momentum correlation time $\tau_{J}$ of simulated heavy and light water as functions of the density $\rho$ in the supercritical region. (b) The average dipole moment $\langle\mu\rangle$ of a water molecule as a function of the density $\rho$. The temperature is $400{ }^{\circ} \mathrm{C}$ when $\rho \leqslant 0.6$, and it is $30^{\circ} \mathrm{C}$ when $\rho=1.0$.

model is in good agreement with the corresponding experimental value by Jonas et al. According to the simulation results in Fig. 2(a), $\tau_{J}^{h} / \tau_{J}^{l} \approx 1$.3. Equation (2) then shows, by virtue of $I^{h} / I^{l} \approx 2$ and $\gamma^{h} / \gamma^{l} \approx 1 / 6.5$, that $1 / T_{1, \mathrm{sr}}^{h}$ is smaller than $1 / T_{1, \mathrm{sr}}^{l}$ by an order of magnitude. Furthermore, since the proton $T_{1}$ of light water is determined by the spin-rotation mechanism in the supercritical conditions, $T_{1, \mathrm{sr}}^{l}$ is essentially equal to $T_{1}$ of light water. ${ }^{22,23,45}$ It is thus implied, in combination with Jonas et al.'s $T_{1}$ data of supercritical light water, that $1 / T_{1, \mathrm{sr}}^{h}$ is an order of magnitude smaller than $1 / T_{1}$ of supercritical heavy water shown in Table I. For example, when the temperature is $400^{\circ} \mathrm{C}$, the contribution of the spinrotation mechanism to the deuteron $1 / T_{1}$ of $\mathrm{D}_{2} \mathrm{O}$ is $\sim 8$ and $\sim 1 \%$ at $\rho=0.1$ and 0.6 , respectively. ${ }^{46}$ Therefore, it is concluded that the deuteron $T_{1}$ of supercritical heavy water is dominated by the quadrupolar mechanism. This conclusion is also valid in the subcritical conditions because the contribution of the spin-rotation mechanism is smaller at a lower temperature.

In the quadrupolar mechanism, the deuteron $T_{1}$ is related to the correlation time $\tau_{2 R}$ through

$$
\frac{1}{T_{1}}=\frac{3 \pi^{2}}{2}\left(\frac{e^{2} Q q}{h}\right)^{2} \tau_{2 R}
$$

where $h$ is the Planck constant and $e^{2} Q q / h$ is the quadrupolar coupling constant (QCC) which represents the interaction between the quadrupole moment of the $\mathrm{D}$ nucleus $(e Q ; Q$ $=2.77 \times 10^{-11} \AA^{2}$ for the $\mathrm{D}$ nucleus) and the electric field gradient at the nucleus $(e q)$. When $\mathrm{D}_{2} \mathrm{O}$ is in the ambient liquid conditions, the QCC is taken to be $256 \mathrm{kHz} \cdot{ }^{47-49}$ When $\mathrm{D}_{2} \mathrm{O}$ is in the dilute gas phase, on the other hand, the
QCC is determined to be $308 \mathrm{kHz}{ }^{47,50}$ This shows that the QCC of $\mathrm{D}_{2} \mathrm{O}$ is dependent on the state of the system. Since a supercritical state lies between the ambient liquid phase and the dilute gas phase, the QCC at the supercritical state is considered to be between 256 and $308 \mathrm{kHz}$. In other words, when the QCC is taken to be equal to 256 and $308 \mathrm{kHz}$, the $\tau_{2 R}$ obtained from Eq. (3) are upper and lower bounds for the exact $\tau_{2 R}$, respectively.

The QCC value is in principle affected by the intermolecular interactions. According to our molecular dynamics simulations, however, the intermolecular contribution to the QCC was typically found to be less than $5 \mathrm{kHz}$, and it is justified to consider that the QCC is dominated by the intramolecular polarization state. In this case, the QCC for the $\mathrm{D}$ nucleus of $\mathrm{D}_{2} \mathrm{O}$ is determined almost entirely by the covalent $\sigma$ electrons in the $\mathrm{O}-\mathrm{D}$ bond. Thus, $\tau_{2 R}$ describes the reorientational relaxation of the unit vector along the $\mathrm{O}-\mathrm{D}$ bond and is expressed as

$$
\tau_{2 R}=\int_{0}^{\infty} d t\left\langle\frac{3}{2} \cos ^{2} \theta(t)-\frac{1}{2}\right\rangle,
$$

where $\theta(t)$ is the angle between the $\mathrm{O}-\mathrm{D}$ bond vectors at time 0 and at time $t$ and $\langle\cdots\rangle$ denotes the ensemble average. The effect of the anisotropy of the electric field gradient is neglected in Eq. (3) since the distribution of the $\sigma$ electrons is almost cylindrical on the O-D axis. ${ }^{47-49}$ It should be noted that $\tau_{2 R}$ is the relaxation time of a single water molecule, while the dielectric relaxation time as reported by Okada et al $^{30,31}$ characterizes the collective motion of all the water molecules in the system. Furthermore, Eq. (3) is a valid expression irrespective of the type of reorientational relaxation and is applicable to a nondiffusive relaxation.

Since the intramolecular polarization state of a water molecule is characterized by its dipole moment, we further assume that the deviation of the QCC value at a given thermodynamic state from that at the dilute gas state is proportional to the corresponding deviation of the average dipole moment of a water molecule. ${ }^{51}$ The average dipole moment of the TIP4P-FQ model at the thermodynamic states of interest is shown in Fig. 2(b) and is used to estimate the QCC value for supercritical water. The approximate QCC value obtained in this procedure is a variable between 256 and 308 $\mathrm{kHz}$ and determines the reorientational correlation time $\tau_{2 R}$ uniquely through Eq. (3). In the supercritical conditions, Table I lists $\tau_{2 R}$ determined from the variable QCC with the upper and lower bounds obtained by setting $\mathrm{QCC}=256$ and $308 \mathrm{kHz}$, respectively. When the temperature is below the critical, on the other hand, Table I lists only $\tau_{2 R}$ estimated by adopting the ambient liquid phase value of $256 \mathrm{kHz}$ for the QCC.

In Fig. 3(a), we show $\tau_{2 R}$ as a function of the density $\rho$ over the thermodynamic states examined in the present work. In this figure, the QCC value adopted in Eq. (3) is the ambient liquid phase value of $256 \mathrm{kHz}$ when $\rho>0.6$ and the system is on the liquid branch of the saturation curve. At the supercritical states with $\rho \leqslant 0.6, \tau_{2 R}$ shown in Fig. 3(a) is determined above from the variable QCC (at $\rho=+0.0$, the effect of the difference between the variable QCC and the dilute gas phase value of $308 \mathrm{kHz}$ is negligible in the precision 

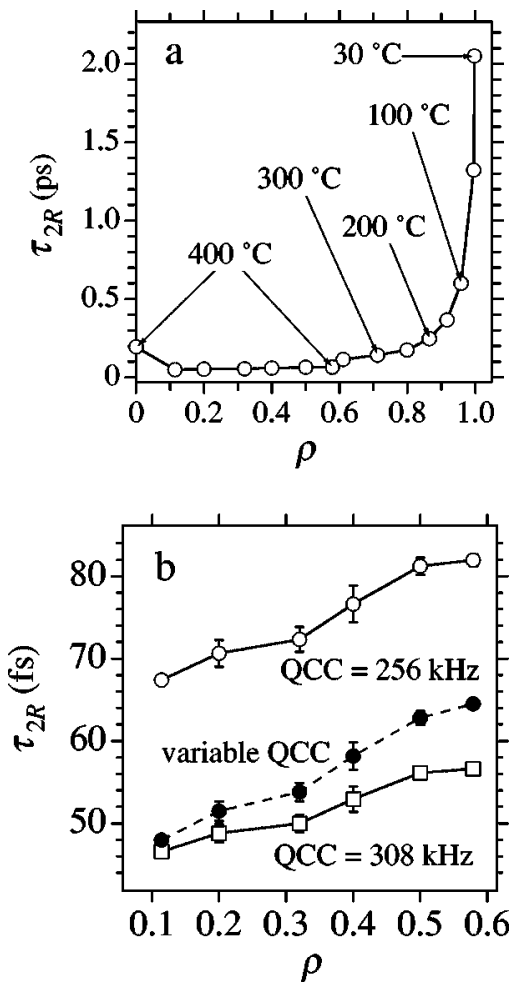

FIG. 3. The reorientational correlation time $\tau_{2 R}$ of heavy water as a function of the density $\rho$ (a) in the whole range examined in this work and (b) in the supercritical region. In (a), the quadrupolar coupling constant (QCC) is taken to be the ambient liquid phase value of $256 \mathrm{kHz}$ when $\rho>0.6$, and it is determined as a variable from the computer simulation results of the average dipole moment when $\rho \leqslant 0.6$. The temperature is $400{ }^{\circ} \mathrm{C}$ when $\rho$ $\leqslant 0.6$, and it is uniquely determined from the liquid branch of the saturation curve when $\rho>0.6$. In (b), the open circles connected by the solid line represent $\tau_{2 R}$ obtained from Eq. (3) by taking QCC $=256 \mathrm{kHz}$, the filled circles connected by the dashed line represent $\tau_{2 R}$ obtained by estimating the variable QCC from the simulations, and the open squares connected by the solid line represent $\tau_{2 R}$ obtained by taking QCC $=308 \mathrm{kHz}$. At each state, upper and lower bounds for $\tau_{2 R}$ are obtained by adopting QCC $=256$ and $308 \mathrm{kHz}$, respectively.

of the figure). It is seen that $\tau_{2 R}$ decreases rapidly with the temperature on the liquid branch of the saturation curve. While the reorientational relaxation proceeds on the order of picosecond in ambient water, it is on the order of several tens of femtoseconds when a supercritical state is realized. In the supercritical conditions with $0.1 \leqslant \rho \leqslant 0.6, \tau_{2 R}$ determined from the variable QCC is plotted in Fig. 3(b) as a function of $\rho$ with the upper and lower bounds obtained by adopting $\mathrm{QCC}=256$ and $308 \mathrm{kHz}$, respectively. At a fixed supercritical temperature of $400{ }^{\circ} \mathrm{C}, \tau_{2 R}$ is between 45 and $80 \mathrm{fs}$ at all the densities in Fig. 3(b). When the chemical shift $\delta$ is concerned in the supercritical conditions, it was found in previous papers that the strength of the density dependence expressed as $\rho \partial \delta / \partial \rho$ is comparable to $\delta$ itself. ${ }^{6,7}$ For the reorientational correlation time $\tau_{2 R}$, in contrast, Fig. 3(b) shows that $\tau_{2 R}$ changes only by $\sim 30 \%$ in response to the density variation from $\rho=0.1$ to 0.6 at a fixed temperature of $400{ }^{\circ} \mathrm{C}$. This shows that the rotational dynamics reflects only partially the change in the state of the hydrogen bonding caused by the density variation. Actually, the "free-rotor correlation time" is $\sim 45 \mathrm{fs}$ at $400^{\circ} \mathrm{C}$ and is comparable to the $\tau_{2 R}$ observed. ${ }^{52-54}$

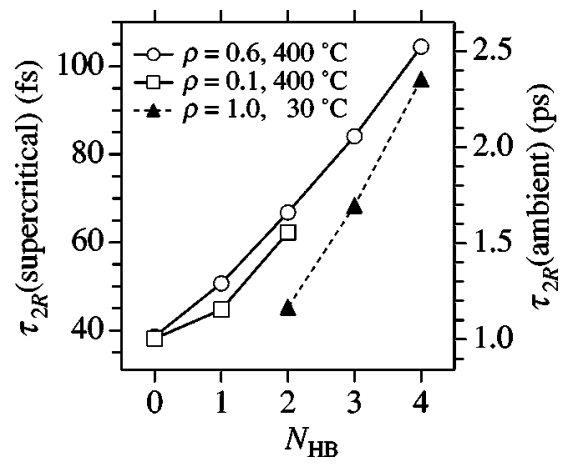

FIG. 4. The reorientational correlation time $\tau_{2 R}$ of simulated heavy water as a function of the number $N_{\mathrm{HB}}$ of hydrogen bonds at supercritical states of $\rho=0.1$ and 0.6 and $400{ }^{\circ} \mathrm{C}$ and at an ambient state of $\rho=1.0$ and $30^{\circ} \mathrm{C}$.

In order to closely see the effect of the hydrogen bonding state on the rotational dynamics, we examine the dependence of $\tau_{2 R}$ on the number of hydrogen bonds at time 0 in Eq. (4) by using the molecular dynamics simulations. Following Ref. 8, we call a pair of water molecules hydrogenbonded when the distance of the closest intermolecular $\mathrm{O}-\mathrm{H}$ (O-D) pair is smaller than the threshold distance $2.4 \AA$. In this definition, the number of hydrogen bonds of a water molecule is obviously four in the ice configuration. We correlate $\tau_{2 R}$ to the number $N_{\mathrm{HB}}$ of hydrogen bonds at the initial time 0 in Eq. (4). Figure 4 then shows $\tau_{2 R}$ of $\mathrm{D}_{2} \mathrm{O}$ as a function of $N_{\mathrm{HB}}$ at supercritical states of $\rho=0.1$ and 0.6 and $400{ }^{\circ} \mathrm{C}$ and at an ambient state of $\rho=1.0$ and $30^{\circ} \mathrm{C}$. It is observed that the absolute change in $\tau_{2 R}$ against the variation of $N_{\mathrm{HB}}$ is larger by orders of magnitude at the ambient state than at the supercritical states. On the other hand, the relative change at the supercritical states is on the average $\sim 2 / 3$ of that at the ambient state. When seen on the relative scale, therefore, the dependence of the rotational dynamics on the hydrogen bonding state in supercritical water is weaker than but is on the same order of magnitude as that in ambient water. ${ }^{55}$ According to Fig. 3(b) and the number $N_{\mathrm{HB}}$ of hydrogen bonds determined in Ref. 7, the experimental $\tau_{2 R}$ increases by $\sim 20 \%$ per hydrogen bond when the density is varied at a constant supercritical temperature. This relative increase is comparable to that observed in Fig. 4 at fixed density and temperature in the supercritical conditions.

According to Fig. 2(a), the angular momentum correlation time $\tau_{J}$ of heavy water is larger by $\sim 30 \%$ than that of light water. It is then inferred from Jonas et al.'s $\tau_{J}$ data of light water ${ }^{22,23}$ that $\tau_{J}$ of heavy water is 50 fs at $\rho=0.6$ and $400{ }^{\circ} \mathrm{C}$ and is larger at a lower density. In this case, the reorientational correlation time shown in Fig. 3 satisfies $\tau_{2 R} \lesssim \tau_{J}$ over the entire range of supercritical conditions examined, as expected by Jonas et al. ${ }^{22,23}$ Thus, it is concluded that the reorientational relaxation is not diffusive and that the inertial effect is operative. The present conclusion is consistent with the molecular dynamics simulation results in Fig. 2(a). Indeed, Fig. 2(a) shows for both supercritical $\mathrm{H}_{2} \mathrm{O}$ and $\mathrm{D}_{2} \mathrm{O}$ that $\tau_{2 R}$ is comparable to or smaller than $\tau_{J}$. Furthermore, the ratios of $\tau_{2 R}$ and $\tau_{J}$ of $\mathrm{D}_{2} \mathrm{O}$ to those of $\mathrm{H}_{2} \mathrm{O}$ in the supercritical conditions are $\sim 1.3$ and are close to the square root of the ratio of the moment of inertia. Since the viscosity 
is experimentally coincident within $\sim 5 \%$ between $\mathrm{H}_{2} \mathrm{O}$ and $\mathrm{D}_{2} \mathrm{O}$ in the supercritical conditions, ${ }^{32}$ the $\tau_{2 R}$ difference between $\mathrm{H}_{2} \mathrm{O}$ and $\mathrm{D}_{2} \mathrm{O}$ reflects the inertial effects in the reorientational relaxation. ${ }^{56-58}$ At the ambient condition of $\rho$ $=1.0$ and $30^{\circ} \mathrm{C}$, on the other hand, since the angular momentum relaxation was found in the molecular dynamics simulations to be faster than the reorientational relaxation by orders of magnitude, the rotational diffusion limit is realized.

In Jonas et al.'s relaxation study of supercritical light water, they evaluated $\tau_{2 R}$ by assuming that it is inversely proportional to the translational diffusion constant. ${ }^{22,23}$ Figure 3(b) and the experimentally determined translational diffusion constant ${ }^{24,59}$ show that Jonas et al.'s approximate procedure is effective to obtain a rough estimate of $\tau_{2 R}$. The procedure overestimates the density dependence of $\tau_{2 R}$ by a factor of $\sim 4$, however, due to the stronger density dependence of the translational diffusion constant. It is then seen in combination with the viscosity data ${ }^{32}$ that the (relative) variation is stronger in the order of the translational diffusion, the viscosity, and the reorientational relaxation when the density is changed at a fixed supercritical temperature. The weaker density dependence of an orientational degree of freedom is evident in the context of the equilibrium structure. In Ref. 8, it was shown that while the radial distribution function of supercritical water involves a higher peak at a lower density, the orientational distribution function is essentially independent of the density variation at a fixed supercritical temperature.

According to Fig. 3, the experimentally determined $\tau_{2 R}$ at the supercritical states is an increasing function of the density $\rho$ in the range $\rho \geq 0.1$ and exhibits an inversion of the $\rho$ dependence in the low-density regime of $\rho \leq 0.1$. This density dependence of $\tau_{2 R}$ is in agreement with the simulation results shown in Fig. 2(a). The agreement is also satisfactory with the results of the molecular dynamics simulations by Yoshii et al. using the RPOL polarizable model ${ }^{60,61}$ and by Balbuena et al. using the SPC/E effective potential model. ${ }^{62,63}$ On the other hand, it was reported by Okada et al. that the dielectric relaxation time exhibits a sharp increase with the density reduction below $\rho=\sim 0.4$, in clear disagreement with recent computer simulation results. ${ }^{30,31,64,65}$ Actually, both the reorientational correlation time $\tau_{2 R}$ obtained from NMR and the dielectric relaxation time are, in principle, to diverge in the limit of zero density because the angular momentum of each molecule is conserved when the intermolecular interactions are absent. The comparison of our $\tau_{2 R}$ with Okada et al.'s dielectric relaxation time further meets the expectation that $\tau_{2 R}$ is smaller than the dielectric relaxation time; while $\tau_{2 R}$ describes the motion of a single molecule in terms of the second-order Legendre polynomial, the dielectric relaxation time represents the collective motion of the molecules in the system in terms of the first-order Legendre polynomial. Nevertheless, the inversion of the density dependence is evidenced at a much higher density for Okada et al.'s dielectric relaxation measurements than for the NMR and simulations. In addition, it was observed from a Raman spectroscopic study by Okazaki et al. that the inversion is evident below half the critical density for supercritical $\mathrm{CF}_{3} \mathrm{H}$ (the fact that $\mathrm{CF}_{3} \mathrm{H}$ involves a weaker intermolecular interaction than water is reflected in the higher inversion density). ${ }^{66}$ Thus, the apparent disagreement is present in the density dependence between the results from the NMR and simulations and those from Okada et al.'s dielectric relaxation measurements performed in the frequency range up to $40 \mathrm{GHz}$.

\section{CONCLUSIONS}

The spin-lattice relaxation time $T_{1}$ of heavy water $\left(\mathrm{D}_{2} \mathrm{O}\right)$ has been measured at super- and subcritical conditions. It has then been shown, in combination with Jonas et al.'s $T_{1}$ data of high-temperature light water $\left(\mathrm{H}_{2} \mathrm{O}\right),{ }^{22,23}$ that the deuteron $T_{1}$ of high-temperature heavy water is dominated by the quadrupolar mechanism and is related to the reorientational correlation time $\tau_{2 R}$ of the $\mathrm{O}-\mathrm{D}$ axis of a single water molecule. According to the experimentally determined $\tau_{2 R}$, the reorientational relaxation proceeds in supercritical water on the order of several tens of femtoseconds, whereas it does on the order of picosecond at ambient conditions. In addition, $\tau_{2 R}$ in supercritical water has been found to be comparable to or smaller than the angular momentum correlation time. In this case, the rotational dynamics is not diffusive and the inertial effect is operative. The relative dependence of $\tau_{2 R}$ on the number of hydrogen bonds in supercritical water has also been studied in combination with molecular dynamics simulations and found to be on the same order of magnitude as that in ambient water. Actually, although $\tau_{2 R}$ is close to the "free-rotor correlation time," it is an increasing function of the density above $\sim 1 / 3$ of the critical.

In order to obtain quantitative insights into a reaction dynamics in supercritical water, it is often necessary to estimate the lifetime of the hydration structure around the reactive species. As noted in the discussion concerning Eq. (1), it is natural to consider for both ionic and nonpolar solutes that when a super- or subcritical condition is realized, the reorientational relaxation of a water molecule around the solute deviates from that in the pure solvent by a factor of less than $\sim 2$. Thus, $\tau_{2 R}$ determined in the present work serves as the characteristic time for the orientational part of a dynamical process in super- and subcritical water. In subsequent work, we treat aqueous solution systems in super- and subcritical conditions and probe the orientational structure around the solute from a dynamical point of view.

\section{ACKNOWLEDGMENTS}

This work is supported by the Research Grant-in-Aid from the Ministry of Education, Science, and Culture (No. 10304047) and by CREST (Core Research for Evolutional Science and Technology) of Japan Science and Technology Corporation (JST). N.M. is also grateful to the Research Grant-in-Aid from the Ministry of Education, Science, and Culture (No. 11740322) and to the Supercomputer Laboratory of Institute for Chemical Research, Kyoto University for generous allocation of computation time. We thank Chihiro Wakai of Kyoto University for insightful discussions and technical advices. We further acknowledge Tsuyoshi Yamaguchi and Yasuo Tsujino of Kyoto University for valuable discussions. 
${ }^{1}$ In this paper, the term "supercritical water" refers to fluid water at a temperature above the critical. Note that the pressure (or density) is not specified.

${ }^{2}$ J. W. Tester, H. R. Holgate, F. J. Armellini et al., in ACS Symposium Series 518, edited by D. W. Tedder and F. G. Pohland (American Chemical Society, Washington, DC, 1993).

${ }^{3}$ R. W. Shaw, T. B. Brill, A. A. Clifford et al., Chem. Eng. News 69, 26 (1991).

${ }^{4}$ J. S. Seewald, Nature (London) 370, 285 (1994).

${ }^{5}$ M. Nakahara, T. Yamaguchi, and H. Ohtaki, Recent Res. Dev. Phys. Chem. 1, 17 (1997).

${ }^{6}$ N. Matubayasi, C. Wakai, and M. Nakahara, Phys. Rev. Lett. 78, 2573 (1997); 78, 4309 (1997).

${ }^{7}$ N. Matubayasi, C. Wakai, and M. Nakahara, J. Chem. Phys. 107, 9133 (1997).

${ }^{8}$ N. Matubayasi, C. Wakai, and M. Nakahara, J. Chem. Phys. 110, 8000 (1999).

${ }^{9}$ E. U. Franck and K. Roth, Discuss. Faraday Soc. 43, 108 (1967).

${ }^{10}$ C. I. Ratcliffe and D. E. Irish, J. Phys. Chem. 86, 4897 (1982).

${ }^{11}$ Y. E. Gorbaty and Y. N. Demianets, Chem. Phys. Lett. 100, 450 (1983).

${ }^{12}$ P. Postorino, R. H. Tromp, M.-A. Ricci et al., Nature (London) 366, 668 (1993).

${ }^{13}$ R. H. Tromp, P. Postorino, G. W. Neilson et al., J. Chem. Phys. 101, 6210 (1994).

${ }^{14}$ K. Yamanaka, T. Yamaguchi, and H. Wakita, J. Chem. Phys. 101, 9830 (1994).

${ }^{15}$ A. K. Soper, F. Bruni, and M. A. Ricci, J. Chem. Phys. 106, 247 (1997).

${ }^{16}$ M.-C. Bellissent-Funel, T. Tassaing, H. Zhao et al., J. Chem. Phys. 107, 2942 (1997)

${ }^{17}$ M. M. Hoffmann and M. S. Conradi, J. Am. Chem. Soc. 119, 3811 (1997).

${ }^{18}$ M. A. Ricci, M. Nardone, A. Fontana et al., J. Chem. Phys. 108, 450 (1998).

${ }^{19}$ S. L. Wallen, B. J. Palmer, and J. L. Fulton, J. Chem. Phys. 108, 4039 (1998).

${ }^{20}$ Y. Ikushima, K. Hatakeda, N. Saito, and M. Arai, J. Chem. Phys. 108, 5855 (1998).

${ }^{21}$ P. Jedlovszky, J. P. Brodholt, F. Bruni et al., J. Chem. Phys. 108, 8528 (1998).

${ }^{22}$ J. Jonas, T. DeFries, and W. J. Lamb, J. Chem. Phys. 68, 2988 (1978).

${ }^{23}$ W. J. Lamb and J. Jonas, J. Chem. Phys. 74, 913 (1981).

${ }^{24}$ W. J. Lamb, G. A. Hoffman, and J. Jonas, J. Chem. Phys. 74, 6875 (1981).

${ }^{25}$ J. Jonas, Science 216, 1179 (1982).

${ }^{26}$ N. Matubayasi and M. Nakahara, J. Chem. Phys. 112, 8089 (2000), and the references cited therein.

${ }^{27}$ C. Wakai and M. Nakahara, J. Chem. Phys. 103, 2025 (1995).

${ }^{28}$ M. Nakahara and C. Wakai, J. Mol. Liq. 65/66, 149 (1995).

${ }^{29}$ M. Nakahara, C. Wakai, Y. Yoshimoto, and N. Matubayasi, J. Phys. Chem. 100, 1345 (1996)

${ }^{30}$ K. Okada, Y. Imashuku, and M. Yao, J. Chem. Phys. 107, 9302 (1997).

${ }^{31}$ K. Okada, M. Yao, Y. Hiejima et al., J. Chem. Phys. 110, 3026 (1999).

${ }^{32}$ Thermophysical Properties of Fluids (The Japan Society of Mechanical Engineers, Tokyo, 1983).

${ }^{33}$ The diameter of a capillary employed in this work may be too large to call our apparatus "capillary." We keep the term "capillary" for the conformity of terminology with Refs. 6 and 7.

${ }^{34}$ Since the $T_{1}$ values from different sizes of capillaries are coincident with each other, the observed $T_{1}$ in this work detects only the water molecules in the bulk. For example, even if a layer of absorbed water is present on the quartz surface, the difference between the $T_{1}$ on the surface and in the bulk does not affect our measurements. In Sec. III, it is shown that the reorientational correlation time determined from the experimental NMR relaxation time is in good agreement with the corresponding simulation result. This provides a further support for the absence of the surface effects.

${ }^{35}$ J. V. Walther and P. M. Orville, Am. Mineral. 68, 731 (1983).

${ }^{36}$ When more than one species are formed from $\mathrm{SiO}_{2}$ in the solution, $\left(1 / T_{1}\right)_{\text {shell }},\left(1 / T_{1}\right)_{\text {complex }}, n, \alpha$, and $m$ in Eq. (1) need to be defined for each species and the sum over the species appears in Eq. (1). Even in this case, the similar argument negates the possibility that the dissolved $\mathrm{SiO}_{2}$ causes an error in the observed $T_{1}$ in the present experimental conditions.

${ }^{37}$ H. G. Hertz, in Water, A Comprehensive Treatise, edited by F. Franks (Plenum, New York, 1973), Vol. 3.

${ }^{38}$ C. E. Manning, Geochim. Cosmochim. Acta 58, 4831 (1994).

${ }^{39}$ The simulation temperature is not required to be identical to the experi- mental temperature for the physics of our interest. When the phenomena essentially determined by the potential energy are concerned, it seems reasonable to adopt the simulation temperature involving the same reduced temperature as the experimental temperature. When the phenomena concerned are largely affected by the kinetic energy, on the other hand, the simulation temperature needs to be close to the experimental temperature. For the rotational dynamics in high-temperature water, both the potential and kinetic contributions will be relevant. In this work, since we extract only the qualitative or semiquantitative features from the simulations, a precise specification of the simulation temperature will not be necessary.

${ }^{40}$ S. W. Rick, S. J. Stuart, and B. J. Berne, J. Chem. Phys. 101, 6141 (1994).

${ }^{41}$ A. Dullweber, B. Leimkuhler, and R. McLachlan, J. Chem. Phys. 107, 5840 (1997).

${ }^{42}$ N. Matubayasi and M. Nakahara, J. Chem. Phys. 110, 3291 (1999).

${ }^{43} \mathrm{~J}$. McConnell, The Theory of Nuclear Magnetic Relaxation in Liquids (Cambridge University Press, Cambridge, 1987).

${ }^{44}$ N. F. Ramsey, Phys. Rev. 87, 1075 (1952).

${ }^{45}$ T. Tsukahara, M. Harada, Y. Ikeda, and H. Tomiyasu, Chem. Lett. 2000, 420 (2000).

${ }^{46}$ The D purity of our samples is more than $99.9 \%$, and each sample contains a trace amount of proton in the form of HDO. For the sample with $\rho=+0.0$, we measured the proton $T_{1}$ of $\mathrm{HDO}$ at $400{ }^{\circ} \mathrm{C}$ and found that $T_{1}=0.55 \mathrm{~s}$. This implies that the contribution of the spin-rotation mechanism to the deuteron $1 / T_{1}$ of $\mathrm{D}_{2} \mathrm{O}$ is $\sim 10 \%$ at $\rho=+0.0$.

${ }^{47}$ D. Lankhorst, J. Schriever, and J. C. Leyte, Ber. Bunsenges. Phys. Chem. 86, 215 (1982).

${ }^{48}$ R. P. W. J. Struis, J. de Bleijser, and J. C. Leyte, J. Phys. Chem. 91, 1639 (1987).

${ }^{49}$ R. Eggenberger, S. Gerber, H. Huber, D. Searles, and M. Welker, J. Chem. Phys. 97, 5898 (1992).

${ }^{50}$ H. Bluyssen, J. Verhoeven, and A. Dynamus, Phys. Lett. 25A, 214 (1967).

${ }^{51}$ When the local electric field is not too strong, both the electronic and nuclear polarizations of a water molecule will be proportional to the local electric field. In this case, when the deviation of the QCC value at a given configuration from that at the dilute gas state is assumed to be proportional to the local electric field, the proportionality relationship can actually be expressed in terms of the QCC and the electronic (or nuclear) polarization. Thus, although our proportionality assumption is concerned only with the QCC and dipole moment, it will be valid in the presence of the nuclear polarization when both the electronic and nuclear polarizations are proportional to the local electric field.

${ }^{52}$ W. A. Steele, Adv. Chem. Phys. 34, 1 (1976).

${ }^{53}$ J. T. Hynes, R. Kapral, and M. Weinberg, J. Chem. Phys. 69, 2725 (1978).

${ }^{54}$ As pointed out in Ref. 53, the "free-rotor correlation time" is simply the characteristic time for the initial decay of the reorientational time correlation function. The actual correlation time of a free rotor is infinite due to the conservation of the angular momentum and has no relationship to the "free-rotor correlation time." It is thus misleading to consider that the reorientational dynamics of supercritical water treated in this work is "free-rotor-like" and is not affected by the intermolecular interactions.

${ }^{55}$ It is a matter of semantics to judge whether or not the hydrogen bonding affects the reorientational dynamics. According to Fig. 4, however, when the effect of the hydrogen bonding is to be called "strong" in the reorientational dynamics in ambient water, it should be called at least "present" in supercritical water.

${ }^{56}$ In our molecular dynamics simulations, the interaction potential is identical between $\mathrm{H}_{2} \mathrm{O}$ and $\mathrm{D}_{2} \mathrm{O}$ and the differences in the dynamical properties of light and heavy water shown in Fig. 2(a) reflect only the difference in the mass (and moments of inertia). It has actually been found, however, that the hydrogen bonding of $\mathrm{D}_{2} \mathrm{O}$ is stronger by $\sim 0.2 \mathrm{kcal} / \mathrm{mol}$ than that of $\mathrm{H}_{2} \mathrm{O}$ (Ref. 57). In order to see the effect of the hydrogen bond strength, we also performed molecular dynamics simulations of $\mathrm{H}_{2} \mathrm{O}$ at supercritical states of $\rho=0.1$ and 0.6 and $400{ }^{\circ} \mathrm{C}$. In these simulations, the original TIP4P model (Ref. 58) and a modified TIP4P-like model with an increased dipole moment were employed by adopting the same simulation conditions as those described in Sec. II B for the TIP4P-FQ model. The original TIP4P model was constructed so that the dipole moment of a water molecule is fixed at $2.18 \mathrm{D}$. In the modified model, the partial charges on the interaction sites were adjusted to give a fixed dipole moment of $2.33 \mathrm{D}$, and the Lennard-Jones parameters were taken to be identical to those of the original TIP4P model. We made this adjustment of the dipole moment in order to increase the hydrogen bond energy by $\sim 0.2$ $\mathrm{kcal} / \mathrm{mol}$. It was then found that the reorientational correlation time $\tau_{2 R}$ is 
larger by $\sim 10 \%$ for the TIP4P-like model with the increased dipole moment. In other words, the strengthened hydrogen bonding by $\sim 0.2 \mathrm{kcal} /$ mol at a fixed mass of the water molecule does not lead to a $\sim 30 \%$ slowdown of the reorientational relaxation, which is realized in Fig. 2(a) by varying the mass at the fixed interaction potential. Therefore, it is expected for the reorientational relaxation of real supercritical water that the effect of the difference in the mass between $\mathrm{H}_{2} \mathrm{O}$ and $\mathrm{D}_{2} \mathrm{O}$ overwhelms the effect of the difference in the hydrogen bond strength.

${ }^{57}$ Y. Marcus and A. Ben-Naim, J. Chem. Phys. 83, 4744 (1985).

${ }^{58}$ W. L. Jorgensen, J. Chandrasekhar, J. D. Madura et al., J. Chem. Phys. 79, 926 (1983).

${ }^{59}$ T. Tassaing and M.-C. Bellissent-Funel, J. Chem. Phys. 113, 3332 (2000).
${ }^{60}$ N. Yoshii, H. Yoshie, S. Miura, and S. Okazaki, J. Chem. Phys. 109, 4873 (1998).

${ }^{61}$ L. X. Dang, J. Chem. Phys. 97, 2659 (1992).

${ }^{62}$ P. B. Balbuena, K. P. Johnston, P. J. Rossky, and J. K. Hyun, J. Phys. Chem. B 102, 3806 (1998).

${ }^{63}$ H. J. C. Berendsen, J. R. Grigera, and T. P. Straatsma, J. Phys. Chem. 91, 6269 (1987).

${ }^{64}$ M. S. Skaf and D. Laria, J. Chem. Phys. 113, 3499 (2000).

${ }^{65}$ C.-N. Yang and H. J. Kim, J. Chem. Phys. 113, 6025 (2000).

${ }^{66}$ S. Okazaki, M. Matsumoto, I. Okada et al., J. Chem. Phys. 103, 8594 (1995). 